\title{
Multiple positive solutions for third-order $p$-Laplacian functional dynamic equations on time scales
}

Da-Bin Wang ${ }^{1,2^{*}}$ and Wen Guan ${ }^{2}$

"Correspondence:

wangdb96@163.com

1School of Mathematics and

Statistics, Central China Normal

University, Wuhan, Hubei 430079,

People's Republic of China

²Department of Applied

Mathematics, Lanzhou University of

Technology, Lanzhou, Gansu

730050, People's Republic of China

\begin{abstract}
In this paper we consider the following boundary value problems for $p$-Laplacian functional dynamic equations on time scales: $\left[\Phi_{p}\left(u^{\Delta \nabla}(t)\right)\right] \nabla+a(t) f(u(t), u(\mu(t)))=0$, $t \in(0, T)_{\mathbf{T}}, u(t)=\varphi(t), t \in[-r, 0]_{\mathbf{T}}, u^{\Delta}(0)=u^{\Delta \nabla}(T)=0, u(T)+B_{0}\left(u^{\Delta}(\eta)\right)=0$. By using the well-known Leggett-Williams fixed point theorem, some existence criteria of at least three positive solutions are established.
\end{abstract}

MSC: $39 \mathrm{~K} 10 ; 34 \mathrm{~B} 15$

Keywords: time scale; $p$-Laplacian functional dynamic equations; boundary value problem; positive solution; fixed point

\section{Introduction}

The theory of dynamic equations on time scales was introduced by Stefan Hilger in 1988 [1]. Let $\mathbf{T}$ be a time scale, i.e., $\mathbf{T}$ is a nonempty closed subset of $\mathbb{R}$. Let $0, T$ be points in $\mathbf{T}$, an interval $[0, T]_{\mathrm{T}}$ denoting a time scale interval, that is, $[0, T]_{\mathbf{T}}:=[0, T] \cap \mathbf{T}$. Other types of intervals are defined similarly.

Recently, there has been much attention paid to the existence of positive solutions for boundary value problems on time scales [2-22] since the theory of dynamic equations on time scales has been a new important mathematical branch (see, for example, [2328]). However, to the best of our knowledge, there is not much concern for $p$-Laplacian functional dynamic equations on time scales $[16,17]$.

For convenience, throughout this paper we denote $\Phi_{p}(s)$ as the $p$-Laplacian operator, i.e., $\Phi_{p}(s)=|s|^{p-2} s, p>1,\left(\Phi_{p}\right)^{-1}=\Phi_{q}, \frac{1}{p}+\frac{1}{q}=1$.

In [4], Anderson, Avery and Henderson considered the following BVP on time scales:

$$
\begin{aligned}
& {\left[\Phi_{p}\left(u^{\Delta}(t)\right)\right]^{\nabla}+c(t) f(u)=0, \quad t \in(a, b)_{\mathrm{T}},} \\
& u(a)-B_{0}\left(u^{\Delta}(v)\right)=0, \quad u^{\Delta}(b)=0,
\end{aligned}
$$

where $v \in(a, b)_{\mathrm{T}}, f \in C_{\mathbf{l d}}([0,+\infty),[0,+\infty)), c \in C_{\mathbf{l d}}([a, b],[0,+\infty))$, and $K_{m} x \leq B_{0}(x) \leq$ $K_{M} x$ for some positive constants $K_{m}, K_{M}$. They established the existence result of at least one positive solution by a fixed point theorem of a cone expansion and a compression of functional type.

In [17], by using a double fixed point theorem due to Avery et al. [29], Song and Xiao considered the existence of at least twin positive solutions to the following $p$-Laplacian

\section{Springer}

(c2014 Wang and Guan; licensee Springer. This is an Open Access article distributed under the terms of the Creative Commons Attribution License (http://creativecommons.org/licenses/by/2.0), which permits unrestricted use, distribution, and reproduction in any medium, provided the original work is properly cited. 
functional dynamic equations on time scales:

$$
\left\{\begin{array}{l}
{\left[\Phi_{p}\left(u^{\Delta \nabla}(t)\right)\right]^{\nabla}+a(t) f(u(t), u(\mu(t)))=0, \quad t \in(0, T)_{\mathrm{T}},} \\
u(t)=\varphi(t), \quad t \in[-r, 0]_{\mathrm{T}}, \\
u^{\Delta}(0)=u^{\Delta \nabla}(T)=0, \quad u(T)+B_{0}\left(u^{\Delta}(\eta)\right)=0,
\end{array}\right.
$$

where $\eta \in(0, \rho(T))_{\mathrm{T}},-r, 0, T \in \mathbf{T}$.

In [30], Zhao, Wang and Ge considered the existence of at least three positive solutions to the following $p$-Laplacian problem:

$$
\begin{aligned}
& {\left[\Phi_{p}\left(u^{\prime}(t)\right)\right]^{\prime}+a(t) f\left(u, u^{\prime}\right)=0, \quad t \in[0,1],} \\
& u^{\prime}(0)=u(1)=0 .
\end{aligned}
$$

The main tool used in [30] is the Leggett-Williams fixed point theorem.

Motivated by the results mentioned above, in this paper, we shall show that the BVP (1.1) has at least three positive solutions by using the Leggett-Williams fixed point theorem [31]. In this article, we always assume that:

$\left(\mathrm{C}_{1}\right) f:[0,+\infty)^{2} \rightarrow(0,+\infty)$ is continuous;

$\left(C_{2}\right) a: \mathbf{T} \rightarrow(0,+\infty)$ is left dense continuous (i.e., $\left.a \in C_{\mathbf{l d}}(\mathbf{T},(0,+\infty))\right)$ and does not vanish identically on any closed subinterval of $[0, T]_{\mathbf{T}}$, where $C_{\mathbf{l d}}(\mathbf{T},(0,+\infty))$ denotes the set of all left dense continuous functions from $\mathbf{T}$ to $(0,+\infty), \min _{t \in[0, T]_{\mathbf{T}}} a(t)=m$, $\max _{t \in[0, T]_{\mathrm{T}}} a(t)=M$, and $m<M$;

$\left(C_{3}\right) \varphi:[-r, 0]_{\mathrm{T}} \rightarrow[0,+\infty)$ is continuous and $r>0$;

(C $\left.\mathrm{C}_{4}\right) \mu:[0, T]_{\mathrm{T}} \rightarrow[-r, T]_{\mathrm{T}}$ is continuous, $\mu(t) \leq t$ for all $t$;

$\left(C_{5}\right) B_{0}(v)$ is for continuous functions defined on $\mathbb{R}$ such that there exist $B \geq 1$ and $A \geq 1$ such that

$$
B x \leq B_{0}(x) \leq A x, \quad \text { for all } \mathbb{R}
$$

Remark 1.1 Insofar as we have time scales, the technique using the Leggett-Williams fixed point theorem in our paper is not the same as in previous works, since the result of our paper is based on the space $E=C_{\mathbf{l d}}^{\Delta}\left([0, T]_{\mathrm{T}}, \mathbb{R}\right)$ with $\|u\|=\max \left\{\max _{t \in[0, T]_{\mathrm{T}}}|u(t)|\right.$, $\left.\max _{t \in[0, T]} \mathrm{T}^{k}\left|u^{\Delta}(t)\right|\right\}$. Furthermore, the novelty in our paper of the hypotheses utilized in the existence theorem is the condition $\left(\mathrm{H}_{4}\right)$ (see Theorem 3.1) where we impose the effect of $a(t)$, so we use the Leggett-Williams fixed point theorem in the space of $E=$ $C_{\mathbf{l d}}^{\Delta}\left([0, T]_{\mathbf{T}}, \mathbb{R}\right)$. On the other hand, the existence of three solutions is important since we think that the more we have the existence of solutions in similar conditions the more interesting it is.

In the remainder of this section we list the following well-known definitions, which can be found in [24-27].

Definition 1.1 For $t<\sup \mathbf{T}$ and $r>\inf \mathbf{T}$, define the forward jump operator $\sigma$ and the backward jump operator $\rho$, respectively,

$$
\sigma(t)=\inf \{\tau \in \mathbf{T} \mid \tau>t\} \in \mathbf{T}, \quad \rho(r)=\sup \{\tau \in \mathbf{T} \mid \tau<r\} \in \mathbf{T}
$$


for all $t, r \in \mathbf{T}$. If $\sigma(t)>t, t$ is said to be right scattered, and if $\rho(r)<r, r$ is said to be left scattered. If $\sigma(t)=t, t$ is said to be right dense, and if $\rho(r)=r, r$ is said to be left dense. If $\mathbf{T}$ has a right scattered minimum $m$, define $\mathbf{T}_{k}=\mathbf{T}-\{m\}$; otherwise set $\mathbf{T}_{k}=\mathbf{T}$. If $\mathbf{T}$ has a left scattered maximum $M$, define $\mathbf{T}^{k}=\mathbf{T}-\{M\}$; otherwise set $\mathbf{T}^{k}=\mathbf{T}$.

Definition 1.2 For $x: \mathbf{T} \rightarrow \mathbb{R}$ and $t \in \mathbf{T}^{k}$, we define the delta derivative of $x(t), x^{\Delta}(t)$, to be the number (when it exists), with the property that, for any $\varepsilon>0$, there is a neighborhood $U$ of $t$ such that

$$
\left|[x(\sigma(t))-x(s)]-x^{\Delta}(t)[\sigma(t)-s]\right|<\varepsilon|\sigma(t)-s|,
$$

for all $s \in U$. For $x: \mathbf{T} \rightarrow \mathbb{R}$ and $t \in \mathbf{T}_{k}$, we define the nabla derivative of $x(t), x^{\nabla}(t)$, to be the number (when it exists), with the property that, for any $\varepsilon>0$, there is a neighborhood $V$ of $t$ such that

$$
\left|[x(\rho(t))-x(s)]-x^{\nabla}(t)[\rho(t)-s]\right|<\varepsilon|\rho(t)-s|,
$$

for all $s \in V$.

If $\mathbf{T}=\mathbb{R}$, then $x^{\Delta}(t)=x^{\nabla}(t)=x^{\prime}(t)$. If $\mathbf{T}=\mathbb{Z}$, then $x^{\Delta}(t)=x(t+1)-x(t)$ is the forward difference operator, while $x^{\nabla}(t)=x(t)-x(t-1)$ is the backward difference operator.

Definition 1.3 If $F^{\triangle}(t)=f(t)$, then we define the delta integral by

$$
\int_{a}^{t} f(s) \triangle s=F(t)-F(a)
$$

If $\Phi^{\nabla}(t)=f(t)$, then we define the nabla integral by

$$
\int_{a}^{t} f(s) \nabla s=\Phi(t)-\Phi(a)
$$

Throughout this papers, we assume that $\mathbf{T}$ is a closed subset of $\mathbb{R}$ with $0 \in \mathbf{T}_{k}$ and $T \in \mathbf{T}^{k}$.

Lemma 1.1 [10] The following formulas hold:

(i) $\left(\int_{a}^{t} f(s) \triangle s\right)^{\Delta}=f(t)$,

(ii) $\left(\int_{a}^{t} f(s) \triangle s\right)^{\nabla}=f(\rho(t))$,

(iii) $\left(\int_{a}^{t} f(s) \nabla s\right)^{\Delta}=f(\sigma(t))$,

(iv) $\left(\int_{a}^{t} f(s) \nabla s\right)^{\nabla}=f(t)$.

\section{Preliminaries}

In this section, we provide some background material from the theory of cones in Banach spaces and we then state the Leggett-Williams fixed point theorem.

Definition 2.1 Let $E$ be a real Banach space. A nonempty, closed, convex set $P \subset E$ is said to be a cone provided the following conditions are satisfied:

(i) if $x \in P$ and $\lambda \geq 0$, then $\lambda x \in P$;

(ii) if $x \in P$ and $-x \in P$, then $x=0$. 
Every cone $P \subset E$ induces an ordering in $E$ given by

$$
x \leq y \quad \text { if and only if } y-x \in P .
$$

Definition 2.2 Let $E$ be a real Banach space and $P \subset E$ be a cone. A function $\alpha: P \rightarrow$ $[0, \infty)$ is called a nonnegative continuous concave functional if $\alpha$ is continuous and

$$
\alpha(t x+(1-t) y) \geq t \alpha(x)+(1-t) \alpha(y)
$$

for all $x, y \in P$ and $t \in[0,1]$.

Let $a, b, c>0$ be constants, $P_{c}=\{x \in P:\|x\|<c\}, P(\alpha, a, b)=\{x \in P: a \leq \alpha(x),\|x\| \leq b\}$.

To prove our main results, we need the following theorem [31].

Theorem 2.1 (Leggett-Williams) Let $A: \bar{P}_{c} \rightarrow \bar{P}_{c}$ be a completely continuous map and $\alpha$ be a nonnegative continuous concave functional on P such that $\alpha(x) \leq\|x\|, \forall x \in \bar{P}_{c}$. Suppose there exist $a, b, d$ with $0<a<b<d \leq c$, such that:

(i) $\{x \in P(\alpha, b, d): \alpha(x)>b\} \neq \varnothing$ and $\alpha(A x)>b$ for all $x \in P(\alpha, b, d)$;

(ii) $\|A x\|<$ a for all $x \in \bar{P}_{a}$;

(iii) $\alpha(A x)>b$, for all $x \in P(\alpha, b, c)$ with $\|A x\|>d$.

Then $A$ has at least three fixed points $x_{1}, x_{2}, x_{3}$ satisfying

$$
\left\|x_{1}\right\|<a, \quad b<\alpha\left(x_{2}\right), \quad\left\|x_{3}\right\|>a, \quad \text { and } \quad \alpha\left(x_{3}\right)<b .
$$

\section{Main result}

In this section we consider the existence of three positive solutions for the problem (1.1).

We say $u$ is concave on $[0, T]_{\mathrm{T}}$ if $u^{\Delta \nabla}(t) \leq 0$ for $t \in[0, T]_{\mathrm{T}^{k} \cap \mathrm{T}_{k}}$.

We note that $u(t)$ is a solution of the problem (1.1) if and only if

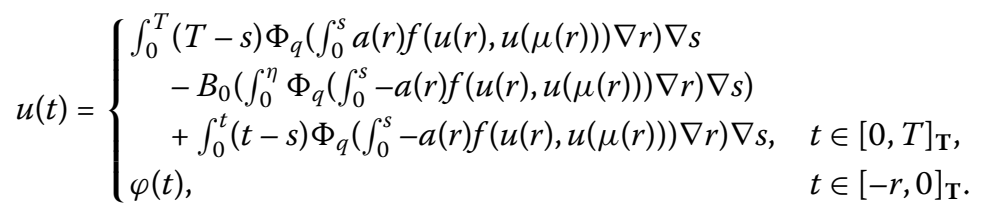

Let $E=C_{\mathbf{l d}}^{\Delta}\left([0, T]_{\mathbf{T}}, \mathbb{R}\right)$ with $\|u\|=\max \left\{\max _{t \in[0, T]_{\mathbf{T}}}|u(t)|, \max _{t \in[0, T]_{T^{k}}}\left|u^{\Delta}(t)\right|\right\}, P=\{u \in$ $E: u$ is nonnegative, decreasing and concave on $\left.[0, T]_{\mathrm{T}}\right\}$. So $E$ is a Banach space with the norm $\|u\|$ and $P$ is a cone in $E$. For each $u \in E$, extend $u(t)$ to $[-r, T]_{\mathrm{T}}$ with $u(t)=\varphi(t)$ for $t \in[-r, 0]_{\mathrm{T}}$.

Define $F: P \rightarrow E$ by

$$
\begin{aligned}
(F u)(t)= & \int_{0}^{T}(T-s) \Phi_{q}\left(\int_{0}^{s} a(r) f(u(r), u(\mu(r))) \nabla r\right) \nabla s \\
& -B_{0}\left(\int_{0}^{\eta} \Phi_{q}\left(\int_{0}^{s}-a(r) f(u(r), u(\mu(r))) \nabla r\right) \nabla s\right) \\
& +\int_{0}^{t}(t-s) \Phi_{q}\left(\int_{0}^{s}-a(r) f(u(r), u(\mu(r))) \nabla r\right) \nabla s, \quad t \in[0, T]_{\mathrm{T}} .
\end{aligned}
$$


It is well known that this operator $F$ is completely continuous.

We seek a fixed point, $u_{1}$, of $F$ in the cone $P$. Define

$$
u(t)= \begin{cases}\varphi(t), & t \in[-r, 0]_{\mathrm{T}}, \\ u_{1}(t), & t \in[0, T]_{\mathrm{T}} .\end{cases}
$$

Then $u(t)$ denotes a positive solution of the problem (1.1).

Lemma 3.1 $F: P \rightarrow P$.

Proof The proof of the lemma is similar to that of $[7,17]$, so we omit it here.

Let $l \in \mathbf{T}$ be fixed such that $0<l<\eta<T$, and set

$$
Y_{1}=\left\{t \in[0, T]_{\mathrm{T}}: \mu(t) \leq 0\right\} ; \quad Y_{2}=\left\{t \in[0, T]_{\mathrm{T}}: \mu(t)>0\right\} ; \quad Y_{3}=Y_{1} \cap[l, \eta]_{\mathrm{T}} .
$$

Throughout this section, we assume $Y_{3} \neq \varnothing$ and $\int_{Y_{3}} a(r) \nabla r>0$.

Now we define the nonnegative continuous concave functional $\alpha: P \rightarrow[0, \infty)$ by

$$
\alpha(u)=\min _{t \in[l, \eta]_{\mathbf{T}}} u(t), \quad \forall u \in P .
$$

It is easy to see that $\alpha(u)=u(\eta) \leq \max _{t \in[0, T]_{\mathrm{T}}}|u(t)| \leq\|u\|$ if $u \in P$ and $\alpha(F u)=(F u)(\eta)$. For convenience, we denote

$$
\rho=T(B+2 T) \Phi_{q}\left(\int_{0}^{T} a(r) \nabla r\right), \quad \delta=A \int_{Y_{3}} \Phi_{q}\left(\int_{0}^{s} a(r) \nabla s\right) \nabla s .
$$

We now state the growth conditions on $f$ so that the problem (1.1) has at least three positive solutions.

Theorem 3.1 Let $0<a<b \leq \frac{A \Phi_{q}\left(\frac{m}{M}\right)}{T(B+2 T)} d<d \leq c$, and suppose that $f$ satisfies the following conditions:

$\left(\mathrm{H}_{1}\right) f(x, \varphi(s))<\Phi_{p}\left(\frac{a}{\rho}\right)$, for all $0 \leq x \leq a$, uniformly in $s \in[-r, 0]_{\mathrm{T}} ; f\left(x_{1}, x_{2}\right)<\Phi_{p}\left(\frac{a}{\rho}\right)$, for all $0 \leq x_{i} \leq a, i=1,2$,

$\left(\mathrm{H}_{2}\right) f(x, \varphi(s)) \leq \Phi_{p}\left(\frac{c}{\rho}\right)$, for all $0 \leq x \leq c$, uniformly in $s \in[-r, 0]_{\mathrm{T}} ; f\left(x_{1}, x_{2}\right) \leq \Phi_{p}\left(\frac{c}{\rho}\right)$, for all $0 \leq x_{i} \leq c, i=1,2$,

$\left(\mathrm{H}_{3}\right) f(x, \varphi(s))>\Phi_{p}\left(\frac{b}{\delta}\right)$, for all $b \leq x \leq d$, uniformly in $s \in[-r, 0]_{\mathrm{T}}$,

$\left(\mathrm{H}_{4}\right) \Phi_{q}\left(\min _{x \in[0, c]} f(x, \varphi(s))\right) \cdot \Phi_{q}\left(\frac{M}{m}\right) \int_{Y_{3}} \Phi_{q}\left(\int_{0}^{s} a(r) \nabla r\right) \nabla s \geq \Phi_{q}\left(\max _{x_{1}, x_{2} \in[0, c]} f\left(x_{1}, x_{2}\right)\right)$. $\Phi_{q}\left(\int_{0}^{T} a(r) \nabla r\right)$, uniformly in $s \in[-r, 0]_{\mathrm{T}}$.

Then the problem (1.1) has at least three positive solutions of the form

$$
u(t)= \begin{cases}\varphi(t), & t \in[-r, 0]_{\mathrm{T}}, \\ u_{i}(t), & t \in[0, T]_{\mathrm{T}}, i=1,2,3,\end{cases}
$$

where $\left\|u_{1}\right\|<a, b<\alpha\left(u_{2}\right),\left\|u_{3}\right\|>a$ and $\alpha\left(u_{3}\right)<b$. 
Proof We first assert that $F: \bar{P}_{c} \rightarrow \bar{P}_{c}$.

Indeed, if $u \in \bar{P}_{c}$, then, in view of Lemma 3.1, we have $F \bar{P}_{c} \subset P$. Furthermore, $\forall u \in \bar{P}_{c}$, we have $0 \leq u \leq c$, and then from $\left(\mathrm{H}_{2}\right)$, we have

$$
\begin{aligned}
|F u(t)|= & \int_{0}^{T}(T-s) \Phi_{q}\left(\int_{0}^{s} a(r) f(u(r), u(\mu(r))) \nabla r\right) \nabla s \\
& -B_{0}\left(\int_{0}^{\eta} \Phi_{q}\left(\int_{0}^{s}-a(r) f(u(r), u(\mu(r))) \nabla r\right) \nabla s\right) \\
& +\int_{0}^{t}(t-s) \Phi_{q}\left(\int_{0}^{s}-a(r) f(u(r), u(\mu(r))) \nabla r\right) \nabla s \\
< & \int_{0}^{T} T \Phi_{q}\left(\int_{0}^{T} a(r) f(u(r), u(\mu(r))) \nabla r\right) \nabla s \\
& +B \int_{0}^{T} \Phi_{q}\left(\int_{0}^{T} a(r) f(u(r), u(\mu(r))) \nabla r\right) \nabla s \\
& +\int_{0}^{T} T \Phi_{q}\left(\int_{0}^{T} a(r) f(u(r), u(\mu(r))) \nabla r\right) \nabla s \\
= & T(B+2 T) \Phi_{q}\left(\int_{Y_{1}} a(r) f(u(r), \varphi(\mu(r))) \nabla r+\int_{Y_{2}} a(r) f(u(r), u(\mu(r))) \nabla r\right) \\
\leq & T(B+2 T) \Phi_{q}\left(\int_{0}^{T} a(r) \nabla r\right) \frac{c}{\rho} \\
= & c, \quad \\
& \leq \frac{c}{B+2 T} \\
& \leq c . \\
\left|(F u)^{\Delta}(t)\right| & =\left|\int_{0}^{t} \Phi_{q}\left(\int_{0}^{s} a(r) f(u(r), u(\mu(r))) \nabla r\right) \nabla s\right| \\
& \leq \int_{0}^{T} \Phi_{q}\left(\int_{0}^{T} a(r) f(u(r), u(\mu(r))) \nabla r\right) \nabla s \\
& =T \Phi_{q}\left(\int_{Y_{1}} a(r) f(u(r), \varphi(\mu(r))) \nabla r+\int_{Y_{2}} a(r) f(u(r), u(\mu(r))) \nabla r\right) \\
&
\end{aligned}
$$

Therefore, $\|F u\| \leq c$, i.e., $F: \bar{P}_{c} \rightarrow \bar{P}_{c}$.

By $\left(\mathrm{H}_{1}\right)$ and in a way similar to above, we arrive at $F: \bar{P}_{a} \rightarrow P_{a}$.

Next, we assert that $\{u \in P(\alpha, b, d): \alpha(u)>b\} \neq \varnothing$ and $\alpha(A u)>b$ for all $u \in P(\alpha, b, d)$.

Let $u=\frac{b+d}{2}$, then $u \in P,\|u\|=\frac{b+d}{2} \leq d$ and $\alpha(u)=\frac{b+d}{2}>b$. That is, $\{u \in P(\alpha, b, d): \alpha(u)>$ $b\} \neq \varnothing$.

Moreover, $\forall u \in P(\alpha, b, d)$, we have $b \leq u(t) \leq d, t \in[0, \eta]_{\mathrm{T}}$, then from $\left(\mathrm{H}_{3}\right)$, we see that

$$
\begin{aligned}
\alpha(F u) & =(F u)(\eta) \\
& =\int_{0}^{T}(T-s) \Phi_{q}\left(\int_{0}^{s} a(r) f(u(r), u(\mu(r))) \nabla r\right) \nabla s
\end{aligned}
$$




$$
\begin{aligned}
& -B_{0}\left(\int_{0}^{\eta} \Phi_{q}\left(\int_{0}^{s}-a(r) f(u(r), u(\mu(r))) \nabla r\right) \nabla s\right) \\
& +\int_{0}^{\eta}(t-s) \Phi_{q}\left(\int_{0}^{s}-a(r) f(u(r), u(\mu(r))) \nabla r\right) \nabla s \\
\geq & -B_{0}\left(\int_{0}^{\eta} \Phi_{q}\left(\int_{0}^{s}-a(r) f(u(r), u(\mu(r))) \nabla r\right) \nabla s\right) \\
\geq & A\left(\int_{0}^{\eta} \Phi_{q}\left(\int_{0}^{s} a(r) f(u(r), u(\mu(r))) \nabla r\right) \nabla s\right) \\
\geq & A\left(\int_{l}^{\eta} \Phi_{q}\left(\int_{0}^{s} a(r) f(u(r), u(\mu(r))) \nabla r\right) \nabla s\right) \\
\geq & A\left(\int_{Y_{3}} \Phi_{q}\left(\int_{0}^{s} a(r) f(u(r), \varphi(\mu(r))) \nabla r\right) \nabla s\right) \\
> & A \int_{Y_{3}} \Phi_{q}\left(\int_{0}^{s} a(r) \nabla s\right) \nabla s \frac{b}{\delta} \\
= & b,
\end{aligned}
$$

as required.

Finally, we assert that $\alpha(F u)>b$, for all $u \in P(\alpha, b, c)$ and $\|F u\|>d$.

To see this, $\forall u \in P(\alpha, b, c)$ and $\|F u\|>d$, then $0 \leq u(t) \leq c, t \in[0, T]_{\mathrm{T}}$, then from $\left(\mathrm{H}_{4}\right)$, we have

$$
\int_{Y_{3}} \Phi_{q}\left(\int_{0}^{s} a(r) f(u(r), u(\mu(r))) \nabla r\right) \nabla s \geq \Phi_{q}\left(\frac{m}{M}\right) \Phi_{q}\left(\int_{0}^{T} a(r) f(u(r), u(\mu(r))) \nabla r\right) .
$$

So,

$$
\begin{aligned}
\alpha(F u)= & (F u)(\eta) \\
= & \int_{0}^{T}(T-s) \Phi_{q}\left(\int_{0}^{s} a(r) f(u(r), u(\mu(r))) \nabla r\right) \nabla s \\
& -B_{0}\left(\int_{0}^{\eta} \Phi_{q}\left(\int_{0}^{s}-a(r) f(u(r), u(\mu(r))) \nabla r\right) \nabla s\right) \\
& +\int_{0}^{\eta}(t-s) \Phi_{q}\left(\int_{0}^{s}-a(r) f(u(r), u(\mu(r))) \nabla r\right) \nabla s \\
\geq & A\left(\int_{0}^{\eta} \Phi_{q}\left(\int_{0}^{s} a(r) f(u(r), u(\mu(r))) \nabla r\right) \nabla s\right) \\
\geq & A\left(\int_{l}^{\eta} \Phi_{q}\left(\int_{l}^{s} a(r) f(u(r), u(\mu(r))) \nabla r\right) \nabla s\right) \\
\geq & A\left(\int_{Y_{3}} \Phi_{q}\left(\int_{0}^{s} a(r) f(u(r), \varphi(\mu(r))) \nabla r\right) \nabla s\right) \\
\geq & A \Phi_{q}\left(\frac{m}{M}\right) \Phi_{q}\left(\int_{0}^{T} a(r) f(u(r), u(\mu(r))) \nabla r\right) \\
= & \frac{A \Phi_{q}\left(\frac{m}{M}\right)}{T(B+2 T)} T(B+2 T) \Phi_{q}\left(\int_{0}^{T} a(r) f(u(r), u(\mu(r))) \nabla r\right) \\
\geq & \frac{A \Phi_{q}\left(\frac{m}{M}\right)}{T(B+2 T)}\|F u\|
\end{aligned}
$$




$$
\begin{aligned}
& >\frac{A \Phi_{q}\left(\frac{m}{M}\right)}{T(B+2 T)} d \\
& \geq b .
\end{aligned}
$$

To sum up, all the hypotheses of Theorem 2.1 are satisfied. Hence $F$ has at least three fixed points, i.e., the problem (1.1) has at least three positive solutions of the form

$$
u(t)= \begin{cases}\varphi(t), & t \in[-r, 0]_{\mathrm{T}}, \\ u_{i}(t), & t \in[0, T]_{\mathrm{T}}, i=1,2,3,\end{cases}
$$

where $\left\|u_{1}\right\|<a, b<\alpha\left(u_{2}\right),\left\|u_{3}\right\|>a$, and $\alpha\left(u_{3}\right)<b$.

\section{Example}

Let $\mathbf{T}=\left[-\frac{3}{4},-\frac{1}{4}\right] \cup\left\{0, \frac{3}{4}\right\} \cup\left\{\left(\frac{1}{2}\right)^{\mathbb{N}_{0}}\right\}$, where $\mathbb{N}_{0}$ denotes the set of all nonnegative integers.

Consider the following $p$-Laplacian functional dynamic equation on the time scale $\mathbf{T}$ :

$$
\left\{\begin{array}{l}
{\left[\Phi_{p}\left(u^{\Delta \nabla}(t)\right)\right] \nabla+a(t)\left[\frac{6 u^{3}(t)}{u^{3}(t)+u^{3}\left(t-\frac{3}{4}\right)+1}+\frac{1}{5}\right]=0, \quad t \in(0,1)_{\mathrm{T}},} \\
u(t)=\varphi(t) \equiv 0, \quad t \in\left[-\frac{3}{4}, 0\right]_{\mathrm{T}}, \\
u^{\Delta}(0)=u^{\Delta \nabla}(T)=0, \quad u(T)+B_{0}\left(u^{\Delta}(\eta)\right)=0,
\end{array}\right.
$$

where $T=1, p=\frac{3}{2}, B=2, A=3, \mu:[0,1]_{\mathrm{T}} \rightarrow\left[-\frac{3}{4}, 1\right]_{\mathrm{T}}$, and $\mu(t)=t-\frac{3}{4}, r=\frac{3}{4}, \eta=\frac{1}{2}, l=\frac{1}{4}$, $f(u, \varphi(s))=\frac{6 u^{3}}{u^{3}+1}+\frac{1}{5}, f\left(u_{1}, u_{2}\right)=\frac{6 u_{1}^{3}}{u_{1}^{3}+u_{2}^{3}+1}+\frac{1}{5}$, and

$$
a(t)= \begin{cases}1, & t \in\left[0, \frac{1}{2}\right]_{\mathrm{T}} \\ -\frac{99}{50} t+\frac{199}{100}, & t \in\left[\frac{1}{2}, 1\right]_{\mathrm{T}} .\end{cases}
$$

We deduce that $Y_{1}=\left[0, \frac{3}{4}\right]_{\mathrm{T}}, Y_{2}=\left(\frac{3}{4}, 1\right]_{\mathrm{T}}, Y_{3}=\left[\frac{1}{4}, \frac{1}{2}\right]_{\mathrm{T}}$. Then $\int_{Y_{3}} \Phi_{q}\left(\int_{0}^{s} a(r) \nabla s\right) \nabla s=\frac{1}{16}$, $\int_{0}^{T} a(r) \nabla r=\int_{0}^{1} a(r) \nabla r=\frac{503}{800}$.

Thus it is easy to see by calculation that $\rho=4\left(\frac{503}{800}\right)^{2}, \delta=\frac{3}{16}$.

Choose $a=\frac{1}{10}, b=1, d=42,000, c=45,000$; then by $M=1, m=\frac{1}{100}$ we have $0<a<b<$ $\frac{A \Phi_{q}\left(\frac{m}{M}\right)}{T(B+2 T)} d<d<c$, and then

$$
\begin{aligned}
& f(u, \varphi(s)) \leq \frac{6}{1,001}+\frac{1}{5} \approx 0.2004<\Phi_{p}\left(\frac{a}{\rho}\right)=\sqrt{\frac{\frac{1}{10}}{4\left(\frac{503}{800}\right)^{2}}} \approx 0.2515,0 \leq u \leq \frac{1}{10}, \text { uniformly in } \\
& s \in[-r, 0]_{\mathrm{T}} ; \\
& f\left(u_{1}, u_{2}\right) \leq \frac{6}{1,002}+\frac{1}{5} \approx 0.2004<\Phi_{p}\left(\frac{a}{\rho}\right)=\sqrt{\frac{\frac{1}{10}}{4\left(\frac{503}{800}\right)^{2}}} \approx 0.2515,0 \leq u_{i} \leq \frac{1}{10}, i=1,2 ; \\
& f(u, \varphi(s))<6.2<\Phi_{p}\left(\frac{c}{\rho}\right)=\sqrt{\frac{45,000}{4\left(\frac{503}{800}\right)^{2}}} \approx 165,0 \leq u \leq 45,000, \text { uniformly in } s \in\left[-\frac{3}{4}, 0\right]_{\mathrm{T}} ; \\
& f\left(u_{1}, u_{2}\right)<6.2<\Phi_{p}\left(\frac{c}{\rho}\right)=\sqrt{\frac{45,000}{4\left(\frac{503}{800}\right)^{2}}} \approx 165,0 \leq u_{i} \leq 45,000, i=1,2 ; \\
& f(u, \varphi(s)) \geq 3.2>\Phi_{p}\left(\frac{b}{\delta}\right)=\sqrt{\frac{16}{3}} \approx 2.31,1 \leq u \leq 42,000, \text { uniformly in } s \in\left[-\frac{3}{4}, 0\right]_{\mathrm{T}} ; \\
& \Phi_{q}\left(\min _{x \in[0, c]} f(x, \varphi(s))\right) \cdot \Phi_{q}\left(\frac{M}{m}\right) \int_{Y_{3}} \Phi_{q}\left(\int_{0}^{s} a(r) \nabla r\right) \nabla s=25>15.4> \\
& \Phi_{q}\left(\max _{x_{1}, x_{2} \in[0, c]} f\left(x_{1}, x_{2}\right)\right) \cdot \Phi_{q}\left(\int_{0}^{T} a(r) \nabla r\right), \text { uniformly in } s \in\left[-\frac{3}{4}, 0\right]_{\mathrm{T}} .
\end{aligned}
$$

Thus by Theorem 3.1, the BVP (4.1) has at least three positive solutions of the form

$$
u(t)= \begin{cases}u_{i}(t), & t \in[0, T]_{\mathrm{T}}, i=1,2,3, \\ \varphi(t), & t \in[-r, 0]_{\mathrm{T}},\end{cases}
$$

where $\left\|u_{1}\right\|<\frac{1}{10}, 1<\alpha\left(u_{2}\right),\left\|u_{3}\right\|>\frac{1}{10}$, and $\alpha\left(u_{3}\right)<1$. 


\section{Competing interests}

The authors declare that they have no competing interests.

\section{Authors' contributions}

The main idea of this paper was proposed by D-BW and D-BW prepared the manuscript initially and WG performed a part of steps of the proofs in this research. All authors read and approved the final manuscript.

\section{Acknowledgements}

The authors thank the referees and the editors for their helpful comments and suggestions. Research was supported by the Postdoctoral fund in China (Grant No. 2013M531717), the Excellent Young Teacher Training Program of Lanzhou University of Techonology (Grant No. Q200907) and Natural Science Foundation of Gansu Province of China (Grant No. 1310RJYA080)

\section{Received: 13 January 2014 Accepted: 28 April 2014 Published: 13 May 2014}

\section{References}

1. Hilger, S: Analysis on measure chains - a unified approach to continuous and discrete calculus. Results Math. 18, 18-56 (1990)

2. Agarwal, RP, Bohner, M, Wong, PJY: Sturm-Liouville eigenvalue problems on time scales. Appl. Math. Comput. 99, 153-166 (1999)

3. Anderson, DR: Solutions to second-order three-point problems on time scales. J. Differ. Equ. Appl. 8, 673-688 (2002)

4. Anderson, DR, Avery, R, Henderson, J: Existence of solutions for a one dimensional $p$-Laplacian on time-scales. J. Differ. Equ. Appl. 10, 889-896 (2004)

5. Anderson, DR, Cabada, A: Third-order right-focal multi-point problems on time scales. J. Differ. Equ. Appl. 12, 919-935 (2006)

6. Akin, E: Boundary value problems for a differential equation on a measure chain. Panam. Math. J. 10, 17-30 (2000)

7. Bian, LH, He, XP, Sun, HR: Multiple positive solutions of $m$-point BVPs for third-order $p$-Laplacian dynamic equations on time scales. Adv. Differ. Equ. 2009, 262857 (2009)

8. Chyan, CJ, Henderson, J: Eigenvalue problems for nonlinear differential equations on a measure chain. J. Math. Anal. Appl. 245, 547-559 (2000)

9. Erbe, L, Peterson, A: Positive solutions for a nonlinear differential equation on a measure chain. Math. Comput. Model. 32(5-6), 571-585 (2000)

10. Han, W, Kang, S: Multiple positive solutions of a nonlinear third-order BVP for a class of $p$-Laplacian dynamic equations on time scales. Math. Comput. Model. 49, 527-535 (2009)

11. Henderson, J: Multiple solutions for $2 m$-th order Sturm-Liouville boundary value problems on a measure chain J. Differ. Equ. Appl. 6, 417-429 (2000)

12. He, ZM: Double positive solutions of three-point boundary value problems for $p$-Laplacian dynamic equations on time scales. J. Comput. Appl. Math. 182, 304-315 (2005)

13. Hong, SH: Triple positive solutions of three-point boundary value problems for $p$-Laplacian dynamic equations on time scales. J. Comput. Appl. Math. 206, 967-976 (2007)

14. Kaufmann, ER, Raffoul, YN: Positive solutions for a nonlinear functional dynamic equation on a time scale. Nonlinear Anal. 62, 1267-1276 (2005)

15. Peterson, AC, Raffoul, YN, Tisdell, CC: Three point boundary value problems on time scales. J. Differ. Equ. Appl. 10, 843-849 (2004)

16. Song, CX, Xiao, CT: Positive solutions for $p$-Laplacian functional dynamic equations on time scales. Nonlinear Anal. 66 1989-1998 (2007)

17. Song, $C X, G a o, X J$ : Positive solutions for third-order $p$-Laplacian functional dynamic equations on time scales. Bound. Value Probl. 2011, 279752 (2011)

18. Su, YH, Li, WT, Sun, HR: Positive solutions of singular $p$-Laplacian PBPs with sign changing nonlinearity on time scales. Math. Comput. Model. 48, 845-858 (2007)

19. Sun, HR, Li, WT: Existence theory for positive solutions to one-dimensional $p$-Laplacian boundary value problems on time scales. J. Differ. Equ. 240, 217-248 (2007)

20. Sun, JP: A new existence theorem for right focal boundary value problems on a measure chain. Appl. Math. Lett. 18 41-47 (2005)

21. Wang, DB: Existence, multiplicity and infinite solvability of positive solutions for $p$-Laplacian dynamic equations on time scales. Electron. J. Differ. Equ. 96, 1-10 (2006)

22. Wang, DB: Three positive solutions of three-point boundary value problems for $p$-Laplacian dynamic equations on time scales. Nonlinear Anal. 68, 2172-2180 (2008)

23. Agarwal, RP, Bohner, M: Quadratic functionals for second order matrix equations on time scales. Nonlinear Anal. 33 675-692 (1998)

24. Agarwal, RP, Bohner, M: Basic calculus on time scales and some of its applications. Results Math. 35, 3-22 (1999)

25. Atici, FM, Guseinov, GS: On Green's functions and positive solutions for boundary value problems on time scales. J. Comput. Appl. Math. 141, 75-99 (2002)

26. Bohner, M, Peterson, A: Dynamic Equations on Time Scales: An Introduction with Applications. Birkhäuser, Boston (2001)

27. Bohner, M, Peterson, A: Advances in Dynamic Equations on Time Scales. Birkhäuser, Boston (2003)

28. Kaymakcalan, B, Lakshmikantham, V, Sivasundaram, S: Dynamical Systems on Measure Chains. Kluwer Academic, Boston (1996)

29. Avery, Rl, Chyan, CJ, Henderson, J: Twin solutions of boundary value problems for ordinary differential equations and finite difference equations. Comput. Math. Appl. 42, 695-704 (2001)

30. Zhao, DX, Wang, HZ, Ge, WG: Existence of triple positive solutions to a class of $p$-Laplacian boundary value problems. J. Math. Anal. Appl. 328, 972-983 (2007)

31. Leggett, RW, Williams, LR: Multiple positive fixed points of nonlinear operators on ordered Banach spaces. Indiana Univ. Math. J. 28, 673-688 (1979) 
10.1186/1687-1847-2014-145

Cite this article as: Wang and Guan: Multiple positive solutions for third-order $p$-Laplacian functional dynamic equations on time scales. Advances in Difference Equations 2014, 2014:145

Submit your manuscript to a SpringerOpen ${ }^{\circ}$ journal and benefit from:

- Convenient online submission

- Rigorous peer review

- Immediate publication on acceptance

- Open access: articles freely available online

- High visibility within the field

- Retaining the copyright to your article

Submit your next manuscript at $\gg$ springeropen.com 